\title{
Nutzen eines intensiven Trainingsprogrammes bei Patienten mit obstruktiver Atemwegskrankheit
}

\author{
M. Hentschel \\ J. Becker \\ H.-J. Lepthin
}

\section{Effects of a High Intensity Training Program on Patients with Chronic Obstructive \\ Airways Disease (COAD)}

\section{Zusammenfassung}

In einer kontrollierten/prospektiven klinischen Studie untersuchten wir 125 Patienten mit chronisch obstruktiver Atemwegskrankheit (Alter 48,2 \pm 11 Jahre, FEV $_{1} 62,7 \pm 13 \%$ des Solls), von denen 84 ein sportmedizinisch orientiertes Trainingsprogramm hoher Intensität auf dem Fahrradergometer absolvierten. Spiroergometrisch wurde die anaerobe Schwelle (AT) bestimmt und daraus die Trainingsbelastung abgeleitet (AT $+40 \%$ der Differenz zur Maximalleistung). Angestrebt wurden im Verlauf von 4 Wochen minimal 22 Trainingseinheiten à 40 Minuten. Bei vergleichbarer Verbesserung der Lungenfunktion durch Optimierung der Medikation und Physiotherapie fand sich nur für die Trainingsgruppe eine statistisch signifikante Verbesserung der maximalen Leistungsfähigkeit $\left(\mathrm{VO}_{2 \max }\right.$ Zunahme um 286,4 vs. $72,4 \mathrm{ml}$ im Kontrollkollektiv, maximale Wattleistung verbessert um 20,0 vs. 5,7 Watt), der Ausdauerleistungsfähigkeit (AT verbessert um 8,4 vs. 5,1 Watt), subjektiver und metabolischer Beanspruchung bei der Trainingsbelastung sowie der Lebensqualität (SF-36).

\section{Abstract}

In a controlled/prospective clinical trial we examined 125 patients with moderate COAD, 84 of which performed a high-intensity training program on cycle ergometers. Methods: All participants (controls $n=39$, training-group $n=84$, age 48.7 versus 48.1 years, $\mathrm{FEV}_{1} 62.3 \%$ vs. $63.1 \%$ of predicted) were examined spiroergometrically (ramp protocol, increment $10 \mathrm{~W} / \mathrm{min}$ ), the anaerobic threshold (AT) determined and a training intensity calculated (AT $+40 \%$ of the difference to peak exercise). Training was carried out on cycle ergometers with 5 training units weekly at 40 minutes each. We aimed at a minimum of 22 training units during 4 weeks. Results: Both groups improved in the lung function at rest $\left(\mathrm{FEV}_{1}+11 \%\right.$ versus $+12 \%$ in the controls). Only for the training group we found a statistically significant improvement in maximum exercise capacity $\left(\mathrm{O}_{2}\right.$ uptake $+286.4 \mathrm{ml}$ vs. $+72.4 \mathrm{ml}$, maximum workrate +20.0 watts vs. $+5.7 \mathrm{~W}$ ), of the anaerobic threshold (AT improved by 8.4 watts vs. $5.1 \mathrm{~W}$ ) as well as in the metabolic load (lactate decreased by $1.3 \mathrm{mmol}$ vs. $0.2 \mathrm{mmol}$, ventilation decreased by $3.8 \mathrm{l} / \mathrm{min}$ vs. $2.3 \mathrm{l} / \mathrm{min}$ ). In most subscales of the SF-36 questionnaire quality of living improved more clearly in the training group, whereas the LAQ questionnaire showed no group-specific differences and no improvement after training. Conclusion: We conclude from the result that a high intensity training program can be recommended as a useful and low-risk component in pulmonary rehabilitation programs.

\section{Einleitung}

Körperliche Belastbarkeit ist für Lebensqualität und Arbeitsfähigkeit unabdingbar, - gerade bei der chronisch-obstruktiven Atemwegskrankheit sind die Patienten jedoch in ihrer Leistungs- fähigkeit oft erheblich eingeschränkt. Dabei besteht zum einen eine Einschränkung durch die Atemwegsobstruktion, zum anderen werden aufgrund der Atemnot bei Anstrengung körperliche Belastungen gemieden, so dass sich zusätzlich eine muskuläre Dekonditionierung entwickelt. 
In vielen Rehabilitationsprogrammen für Atemwegspatienten sind daher Trainingselemente enthalten, wobei für den zeitlichen Umfang des Trainings und die Trainingsintensität keine klaren Vorgaben existieren [1,2]. Aus der Sportmedizin werden für Normalpersonen Trainingseinheiten von 30-45 min Dauer, 3-5 mal die Woche, für minimal 4-5 Wochen gefordert, um am Muskel physiologische Veränderungen zu erzielen [3]. Dabei werden Belastungsintensitäten von ca. 70\% der Maximalleistung empfohlen. Bei Atemwegspatienten wird bezüglich der Trainingsintensität zumeist als Untergrenze die Wattzahl an der nach der V-slope-Methode bestimmten respiratorischen anaeroben Schwelle (AT) angesetzt [4], wobei sich nach vorliegenden Arbeiten (insbesondere [5]), größere Erfolge bei höheren Trainingsintensitäten zeigen. Erwartet wird dabei durch ein Training hoher Intensität insbesondere eine Abnahme der Lactazidose bei identischer Belastung, woraus nach Training eine Verbesserung der Atemökonomie und Verminderung der Atemarbeit resultieren soll. Die Notwendigkeit einer hohen Trainingsintensität ist allerdings nicht unbestritten [6] und es sind vornehmlich im ambulanten Bereich auch Steigerungen der Leistungsfähigkeit bei deutlich niedrigerer Trainingsintensität nachgewiesen [7], wobei hier ein Signaleffekt des Trainings bezüglich einer gesunden Lebensführung postuliert wird. Weiterhin scheint es möglich, dass regelmäßiges Training günstige Auswirkungen auf die Immunitätslage hat und so eventuell die Häufigkeit von Atemwegsinfekten vermindert [8]. Da die bisher publizierten Studien meist kleine Fallzahlen aufweisen, war es Ziel der Studie, anhand eines größeren Patientenkollektives den Effekt eines sportmedizinisch orientierten Trainingsprogrammes auf kardiopulmonale Basisparameter zu überprüfen. Eine weitere Fragestellung war, ob im Rahmen des deutschen Systems der Rehabilitation mit relativ kurzer Dauer der stationären Rehabilitation überhaupt nachweisbare Trainingseffekte erzielt werden können.

Da bekannterweise physiologische Funktionsparameter nur schwach mit der Befindlichkeit der Patienten korrelieren, haben wir gleichzeitig die subjektive Einschätzung der Anstrengung abgefragt und zusätzlich mit Fragebögen zur Lebensqualität den Effekt des Trainingsprogrammes überprüft.

\section{Methodik}

\section{Probandenkollektiv}

Bei den Teilnehmern dieser Studie handelte es sich um insgesamt 125 Patienten (17 Frauen, 108 Männer, Alter 48,2 \pm 11 Jahre), die mit einer klinisch stabilen obstruktiven Atemwegserkrankung (relative Einsekundenkapazität $\leq 65 \%$ ) konsekutiv zur stationären Rehabilitation eingewiesen wurden. Die Zuweisung selbst war von uns nicht beeinflussbar, so dass strukturelle systematische Einflüsse unsererseits auf das Probandenkollektiv auszuschließen sind. Für die Teilnahme an der Studie wurde die Fähigkeit gefordert, bei einer Spiroergometrie mit einem Belastungsanstieg von 10 Watt/Minute eine leichte Laktazidose (mindestens $3,0 \mathrm{mmol} / \mathrm{l}$ ) zu erreichen, sowie eine Steady-State-Spiroergometrie mit der errechneten Trainingsintensität für 20 Minuten zu tolerieren.
Ausschlusskriterien waren Infektexazerbationen in den letzten 6 Wochen, relevante Erkrankungen des Herz-Kreislauf-Systemes, trainingsbeeinträchtigende orthopädische Erkrankungen sowie Sprachprobleme (aufgrund der dann fehlenden Auswertbarkeit der Scores zur Lebensqualität). Die Probanden wurden vor Beginn des Trainings über das Ziel der Studie aufgeklärt und gaben ihre schriftliche Einwilligung.

\section{Testprotokolle}

Bei den potenziellen Studienteilnehmern wurde zunächst eine Spiroergometrie mit rampenförmigem Belastungsanstieg durchgeführt. Dabei wurde nach einer Ruhephase von 2-3 Minuten die Belastung um 10 Watt/Minute kontinuierlich gesteigert bis zum Erreichen der Ausbelastungsherzfrequenz (200 - Lebensalter, nach Löllgen [9] ) oder bis zum mitarbeitsbedingten Abbruch. Laktatbestimmungen erfolgten in Ruhe, im Bereich der anaeroben Schwelle sowie eine Minute nach Abbruch; Blutgasanalysen erfolgten in Ruhe und bei Abbruch. Aus der erreichten Wattleistung an der respiratorischen anaeroben Schwelle und der maximalen Wattleistung errechneten wir (in Annäherung an [5]) dann die Trainingsbelastung nach folgender Formel:

Trainingsbelastung $=$ Wattleistung an der anaeroben Schwelle + $40 \%$ der Differenz zur Maximalleistung.

Am zweiten Tag wurde mit der errechneten Trainingsbelastung, wiederum nach 3-minütiger Ruhephase eine Steady-State-Spiroergometrie mit 20 Minuten Dauer durchgeführt, mit Laktatbestimmung in Ruhe und nach 15 Minuten Belastung sowie Blutgasanalysen in Ruhe und nach 15 Minuten. Weitere Zusatzuntersuchungen (Belastungs-EKG, Echokardiographie, CO-Diffusion) stellten sicher, dass das Trainingsprogramm ohne Risiko für die Patienten durchlaufen werden konnte.

\section{Trainingsprotokoll}

Die in die Studie aufgenommenen Patienten wurden alternierend in Trainings- und Kontrollgruppe aufgeteilt. Da wir für die Trainingsgruppe mit einer hohen Abbrecherquote rechneten, haben wir die konsekutiv aufgenommenen Patienten jeweils 8 Wochen für die Trainingsgruppe rekrutiert, dann 4 Wochen für die Kontrollgruppe und ungleiche Gruppengrößen in Kauf genommen. Alle Patienten haben unsere etablierte Rehabilitation durchlaufen, mit Patientenschulung, Optimierung der medikamentösen Therapie (Abb.1), Atemgymnastik, physikalischer Therapie sowie gegebenenfalls Diätberatung oder Nichtrauchertrai-

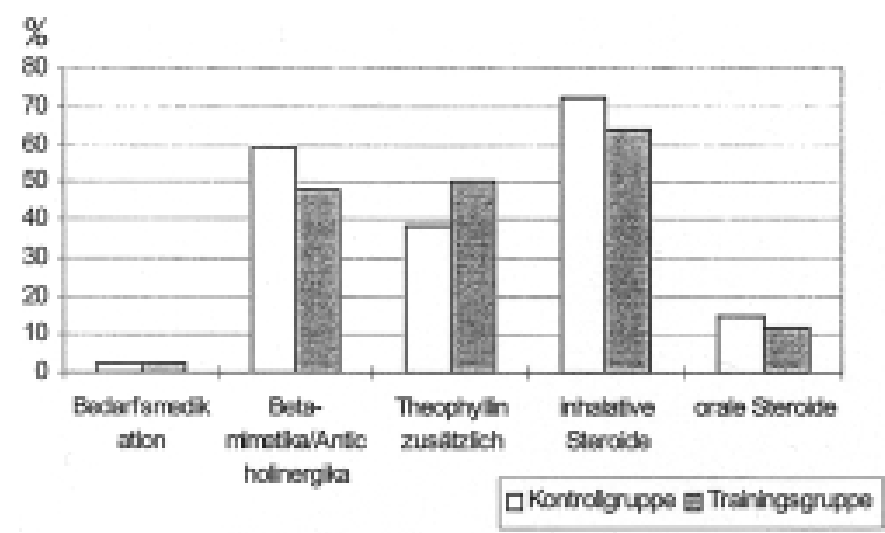

Abb. 1 Medikation nach Gruppen. Dargestellt in Prozent der Fälle. 
ning. Die Interventionsgruppe nahm zusätzlich an einem intensiven Trainingsprogramm teil, welches 5-mal pro Woche 40-minütige Trainingseinheiten mit der oben angegebenen Trainingsbelastung auf dem Fahrradergometer beinhaltete. Alternativ waren in der Eingangsphase auch pro Tag zwei 20-minütige Trainingseinheiten möglich. Angestrebt wurden im Verlauf der Rehabilitation minimal 22 Trainingseinheiten.

\section{Erfassung der Lebensqualität}

Da aus vielfältigen Studien bekannt ist, dass gerade die Beurteilung des Symptoms „Dyspnoe“ nicht nur von physiologischen Parametern abhängig ist, wurde nach jeder Belastungsuntersuchung die subjektiv empfundene Anstrengung mittels der modifizierten Borg-Skala abgefragt [10]. Weiterhin wurde die subjektive Einschätzung von Lebensqualität und Leistungsfähigkeit mittels Fragebogen erfasst. Es wurden bereits validierte Fragebogen verwendet, der MOS-SF 36 („Medical Outcome Study-Short Form “) in der deutschen Übersetzung von Bullinger und der LAQ („Living with Asthma Questionnaire“/Hyland 1991) in der deutschen Version mit 40 Fragen (Fragebogen zur Lebensqualität bei Asthma/Lötfering et al. 1993). Der Fragebogen zur Lebensqualität bei Asthma ist bereits 1994 an Patienten mit chronisch obstruktiver Atemwegskrankheit erprobt worden und eine neuere Arbeit [11] empfiehlt den LAQ auch für den Einsatz bei COPDPatienten. Diese Fragebogen wurden vor und nach Training sowie nach 6 und 12 Monaten von den Studienteilnehmern ausgefüllt.

\section{Statistik}

Die statistische Analyse erfolgte auf dem Personalcomputer mit dem Programm SPSS für Windows. Die Untersuchungsparameter vor und nach dem Training wurden mittels gepaartem t-Test untersucht, für die Gruppenvergleiche wurden ungepaarte t-Tests verwendet. Die Beziehungen zwischen den Variablen wurden mittels linearer Regression und Pearsons Korrelationskoeffizient untersucht. Bei p $<0,05$ wurden Signifikanzen angenommen.

\section{Ergebnisse}

Als Ergebnis der stationären Rehabilitation mit Therapieoptimierung nach den Richtlinien der deutschen Atemwegsliga, Atemschulungsprogramm und begleitender Physiotherapie zeigte sich bei den 125 teilnehmenden Patienten eine statistisch signifikante Verbesserung der Einsekundenkapazität (Tab. 1).

Die Gruppen waren nach Alter, Lungenfunktion, Belastbarkeit und Medikation gut vergleichbar, signifikante geschlechtsbezogene Unterschiede fanden sich nicht. Das von der Interventionsgruppe durchgeführte Trainingsprogramm verlief ohne Komplikationen. Zwei Patienten brachen ab, einmal wegen Infektexazerbation, einmal wegen vorzeitiger Entlassung. Ein Studienteilnehmer weigerte sich nach anfänglicher Zustimmung später die Fragebögen auszufüllen.

\section{Kardiopulmonale Funktionsparameter}

Es wurde bei Anfang und Ende des Trainingsprogramms jeweils eine spiroergometrische Untersuchung mit rampenförmigem Belastungsanstieg (10 Watt/Minute) und mit einem Steady-State
Tab. 1 Funktionsdaten des Untersuchungskollektives vor und nach Rehabilitation. $\mathrm{n}=125,2$ Abbrecher

\begin{tabular}{|c|c|c|c|c|}
\hline & \multicolumn{2}{|c|}{$\begin{array}{l}\text { Kontrollgruppe } n=39 \\
32 \text { männlich, } 7 \text { weiblich }\end{array}$} & \multicolumn{2}{|c|}{$\begin{array}{l}\text { Trainingsgruppe } n=84 \\
74 \text { männlich, } 10 \text { weiblich }\end{array}$} \\
\hline & vor & nach & vor & nach \\
\hline Alter, Jahre & $48,7 \pm 11,9$ & & $48,1 \pm 10,5$ & \\
\hline $\begin{array}{l}\text { FEV }_{1} \text {, Prozent des } \\
\text { Solls }\end{array}$ & $62,3 \pm 13,2$ & $75,4 \pm 16,0^{*}$ & $63,0 \pm 13,4$ & $75,2 \pm 15,9^{*}$ \\
\hline $\begin{array}{l}\text { Residualvolumen } \\
\text { in \% der TLC }\end{array}$ & $47,6 \pm 10,0$ & $44,5 \pm 8,7^{*}$ & $45,8 \pm 8,8$ & $43,3 \pm 9,2^{*}$ \\
\hline $\begin{array}{l}\text { Bodymass Index, } \\
\mathrm{m}^{2} / \mathrm{kg}\end{array}$ & $27,2 \pm 3,9$ & $26,7 \pm 3,3^{*}$ & $27,6 \pm 4,5$ & $27,0 \pm 3,8^{*}$ \\
\hline $\begin{array}{l}\text { errechnete Trainings- } \\
\text { belastung, Watt }\end{array}$ & $80,3 \pm 20,9$ & & $84,5 \pm 21,4$ & \\
\hline
\end{tabular}

${ }^{*}$ signifikant bei $\mathrm{p}<0,01$

Tab. 2 Maximalwerte der Rampenspiroergometrie

\begin{tabular}{|c|c|c|c|c|}
\hline & \multicolumn{2}{|c|}{ Kontrollgruppe $n=39$} & \multicolumn{2}{|c|}{ Interventionsgruppe $n=84$} \\
\hline & vor & nach & vor & nach \\
\hline Leistung Watt & $113,3 \pm 28,5$ & $119,0 \pm 37,0$ & $117,3 \pm 33,1$ & $137,3 \pm 38,5^{* *}$ \\
\hline $\begin{array}{l}\text { Herzfrequenz } \\
\min ^{-1}\end{array}$ & $137,3 \pm 17,7$ & $137,7 \pm 20,5$ & $137,1 \pm 16,0$ & $143,3 \pm 16,6^{* *}$ \\
\hline $\begin{array}{l}\text { Laktat } \\
\mathrm{mmol} / \text { Liter }\end{array}$ & $5,1 \pm 1,8$ & $5,4 \pm 2,3$ & $5,2 \pm 1,7$ & $6,1 \pm 2,0^{* *}$ \\
\hline $\begin{array}{l}\text { Atemminuten- } \\
\text { volumen } 1 / \text { min }\end{array}$ & $53,7 \pm 10,6$ & $55,9 \pm 16,2$ & $56,9 \pm 13,7$ & $64,4 \pm 16,5^{* *}$ \\
\hline $\begin{array}{l}\text { Sauerstoff- } \\
\text { aufnahme } \\
\mathrm{ml} / \mathrm{min} / \mathrm{kg}\end{array}$ & $21,1 \pm 4,7$ & $21,9 \pm 5,5$ & $21,6 \pm 5,4$ & $25,4 \pm 6,7^{* *}$ \\
\hline $\begin{array}{l}\text { Atemfrequenz } \\
\mathrm{min}^{-1}\end{array}$ & $26,6 \pm 5,9$ & $26,0 \pm 5,3$ & $25,9 \pm 5,1$ & $26,9 \pm 5,5^{*}$ \\
\hline
\end{tabular}

${ }^{*}$ signifikant bei $\mathrm{p}<0,05,{ }^{* *}$ signifikant bei $\mathrm{p}<0,01$

Tab. 3 Werte an der anaeroben Schwelle

\begin{tabular}{|c|c|c|c|c|}
\hline & \multicolumn{2}{|c|}{ Kontrollgruppe $n=39$} & \multicolumn{2}{|c|}{ Interventionsgruppe $n=84$} \\
\hline & vor & nach & vor & nach \\
\hline Leistung Watt & $58,6 \pm 20,1$ & $63,7 \pm 15,9$ & $62,4 \pm 16,6$ & $70,8 \pm 18,2^{* *}$ \\
\hline $\begin{array}{l}\text { Herzfrequenz } \\
\text { min }^{-1}\end{array}$ & $108 \pm 12$ & $107 \pm 16$ & $109 \pm 13$ & $110 \pm 12$ \\
\hline $\begin{array}{l}\text { Atemminuten- } \\
\text { volumen } 1 / \text { min }\end{array}$ & $29,6 \pm 6,8$ & $30,5 \pm 6,2$ & $32,2 \pm 6,4$ & $33,9 \pm 6,5^{*}$ \\
\hline $\begin{array}{l}\text { Atemfrequenz } \\
\mathrm{min}^{-1}\end{array}$ & $19,3 \pm 4,6$ & $19,8 \pm 4,0$ & $19,4 \pm 4,9$ & $19,3 \pm 4,7$ \\
\hline $\begin{array}{l}\text { Sauerstoff- } \\
\text { aufnahme } \\
\mathrm{ml} / \mathrm{min} / \mathrm{kg}\end{array}$ & $12,9 \pm 3,0$ & $13,3 \pm 2,4$ & $13,7 \pm 3,0$ & $15,4 \pm 3,2^{* *}$ \\
\hline
\end{tabular}

${ }^{*}$ signifikant bei $\mathrm{p}<0,05,{ }^{* *}$ signifikant bei $\mathrm{p}<0,01$

Protokoll (individuelle Trainingsbelastung) durchgeführt. Verglichen werden aus den bei Anfang und Ende des Trainingsprogrammes durchgeführten Spiroergometrien mit rampenförmiger Belastung die Maximalwerte (Tab. 2) und die Werte an der respiratorischen anaeroben Schwelle (Tab.3), aus den Steady- 
Tab. 4 Messgrößen aus der 15. Minute der Steady-State-Belastung

\begin{tabular}{|c|c|c|c|c|}
\hline & \multicolumn{2}{|c|}{ Kontrollgruppe $n=39$} & \multicolumn{2}{|c|}{ Interventionsgruppe $n=84$} \\
\hline & vor & nach & vor & nach \\
\hline $\begin{array}{l}\text { Laktat mmol/ } \\
\text { Liter }\end{array}$ & $4,5 \pm 2,2$ & $4,4 \pm 1,4$ & $5,0 \pm 1,3$ & $3,7 \pm 1,2^{* *}$ \\
\hline $\begin{array}{l}\text { Atemminuten- } \\
\text { volumen I/min }\end{array}$ & $46,5 \pm 10,9$ & $44,2 \pm 10,6$ & $49,0 \pm 9,9$ & $45,3 \pm 10,0^{* *}$ \\
\hline $\begin{array}{l}\text { Atemfrequenz } \\
\min ^{-1}\end{array}$ & $24,9 \pm 5,2$ & $24,1 \pm 5,2$ & $24,9 \pm 5,5$ & $22,6 \pm 5,5^{* *}$ \\
\hline $\begin{array}{l}\text { Sauerstoff- } \\
\text { aufnahme } \\
\mathrm{ml} / \mathrm{min} / \mathrm{kg}\end{array}$ & $17,6 \pm 4,0$ & $17,0 \pm 3,7$ & $18,6 \pm 4,5$ & $18,5 \pm 4,3$ \\
\hline $\begin{array}{l}\text { Herzfrequenz } \\
\text { min }^{-1}\end{array}$ & $135 \pm 21$ & $127 \pm 19^{* *}$ & $134 \pm 17$ & $125 \pm 14^{* *}$ \\
\hline
\end{tabular}

*signifikant bei $\mathrm{p}<0,05,{ }^{*}$ signifikant bei $\mathrm{p}<0,01$

State-Belastungen die Werte der 15. Belastungsminute (Tab.4). Typische Ergebnisse eines Trainingsprogrammes sind die für die Interventionsgruppe gesteigerte Maximalleistung und maximale Sauerstoffaufnahme (Tab.2), ferner hat sich für die Interventionsgruppe die respiratorische anaerobe Schwelle signifikant erhöht (Tab. 3). In der Steady-State-Untersuchung vor/nach Rehabilitation (Tab.4) fällt als deutlichster Effekt des Trainingsprogrammes die (statistisch signifikant) verminderte Laktatproduktion der Interventionsgruppe bei gleicher vorgegebener Belastung auf. Entsprechend vermindert sich in der Interventionsgruppe auch der Atemaufwand (Atemfrequenz und Atemminutenvolumen) statistisch signifikant. Die Belastungsherzfrequenz fällt sowohl in der Kontroll- als auch in der Interventionsgruppe nach der Rehabilitation signifikant ab.

\section{Subjektive Belastungseinschätzung/Auswertung der Lebens- qualitätsfragebogen}

Die gesteigerte Belastungstoleranz der Interventionsgruppe zeigt sich auch in der Einschätzung der Anstrengung nach der modifizierten Borg-Skala, wobei nach Training identische Belastungen als weniger anstrengend empfunden wurden (Tab. 5).

Vor und nach der Rehabilitation sowie nach 6 und 12 Monaten haben die Studienteilnehmer Fragebogen zur Lebensqualität ausgefüllt. Die Rücklaufquoten waren erstaunlich hoch: nach 6 Monaten Trainingsgruppe 83\%, Kontrollgruppe 92\%, nach 12 Monaten Trainingsgruppe 74\%, Kontrollgruppe 85\%.

Die Auswertung des MOS SF-36 ergibt für die Interventionsgruppe zum Abschluss der Rehabilitation statistisch signifikante Verbesserungen in den Subskalen körperliche Funktionsfähigkeit, körperliche Rollenfunktion, allgemeines Gesundheitsempfinden, Vitalität, psychisches Wohlbefinden und beobachtete Gesundheitsveränderung. Für die Kontrollgruppe finden sich Verbesserungen in den Skalen körperliche Rollenfunktion und Vitalität. Nach sechs Monaten ist nur noch der Wert für körperliche Rollenfunktion der Interventionsgruppe verbessert, nach 12 Monaten sind in beiden Gruppen keine statistisch signifikanten Verbesserungen mehr nachweisbar (Tab. 6 u. 7). In der Auswertung des LAQ-Fragebogens (Tab. 8) zeigt die Interventionsgruppe zum
Tab. 5 Subjektive Belastungseinschätzung nach der modifizierten Borg-Skala (Mittelwerte der Gruppen)

\begin{tabular}{|c|c|c|c|c|}
\hline & \multicolumn{2}{|c|}{ Kontrollgruppe $n=39$} & \multicolumn{2}{|c|}{$\begin{array}{l}\text { Interventionsgruppe } \\
n=84\end{array}$} \\
\hline & vor & nach & vor & nach \\
\hline $\begin{array}{l}\text { Borg-Wert } \\
\text { Rampenbelastung }\end{array}$ & $5,0 \pm 2,1$ & $5,1 \pm 1,8$ & $4,8 \pm 1,5$ & $4,3 \pm 1,6^{*}$ \\
\hline $\begin{array}{l}\text { Borg-Wert } \\
\text { Steady-State-Belastung }\end{array}$ & $5,4 \pm 1,9$ & $5,1 \pm 2,1$ & $4,8 \pm 1,6$ & $3,5 \pm 1,3 * *$ \\
\hline
\end{tabular}

*signifikant bei $\mathrm{p}<0,05,{ }^{* *}$ signifikant bei $\mathrm{p}<0,01$

Tab. 6 MOS SF-36 vor/nach stationärer Rehabilitation

\begin{tabular}{|c|c|c|c|c|}
\hline & \multicolumn{2}{|c|}{ Kontrollgruppe $n=39$} & \multicolumn{2}{|c|}{ Interventionsgruppe $n=84$} \\
\hline & vor & nach & vor & nach \\
\hline $\begin{array}{l}\text { körperliche Funk- } \\
\text { tionsfähigkeit }\end{array}$ & $67,5 \pm 23,3$ & $65,9 \pm 26,2$ & $68,8 \pm 21,2$ & $74,9 \pm 22,1^{*}$ \\
\hline $\begin{array}{l}\text { körperliche } \\
\text { Rollenfunktion }\end{array}$ & $31,8 \pm 39,8$ & $45,9 \pm 43,9^{*}$ & $47,2 \pm 41,6$ & $64,9 \pm 42,3^{* *}$ \\
\hline $\begin{array}{l}\text { körperliche } \\
\text { Schmerzen }\end{array}$ & $54,1 \pm 25,6$ & $65,1 \pm 28,1$ & $58,9 \pm 29,5$ & $65,6 \pm 28,0$ \\
\hline $\begin{array}{l}\text { allgemeine } \\
\text { Gesundheits- } \\
\text { wahrnehmung }\end{array}$ & $51,6 \pm 18,6$ & $49,4 \pm 19,5$ & $44,1 \pm 18,2$ & $50,2 \pm 20,5^{* *}$ \\
\hline Vitalität & $51,4 \pm 20,2$ & $57,8 \pm 18,9^{*}$ & $50,1 \pm 20,6$ & $58,6 \pm 19,9^{* *}$ \\
\hline $\begin{array}{l}\text { soziale Funktions- } \\
\text { fähigkeit }\end{array}$ & $51,0 \pm 14,9$ & $44,4 \pm 11,1$ & $49,2 \pm 10,0$ & $49,4 \pm 10,8$ \\
\hline $\begin{array}{l}\text { emotionale } \\
\text { Rollenfunktion }\end{array}$ & $58,3 \pm 45,3$ & $72,8 \pm 41,6$ & $67,1 \pm 41,8$ & $74,3 \pm 41,0$ \\
\hline $\begin{array}{l}\text { psychisches } \\
\text { Wohlbefinden }\end{array}$ & $69,3 \pm 20,1$ & $73,7 \pm 15,2$ & $66,2 \pm 21,2$ & $74,9 \pm 17,4^{* *}$ \\
\hline $\begin{array}{l}\text { beobachtete } \\
\text { Gesundheits- } \\
\text { veränderung }\end{array}$ & $3,1 \pm 1,1$ & $2,4 \pm 1,1^{* *}$ & $3,0 \pm 1,0$ & $2,4 \pm 1,1^{* *}$ \\
\hline
\end{tabular}

*signifikant bei $\mathrm{p}<0,05,{ }^{* *}$ signifikant bei $\mathrm{p}<0,01$

Abschluss der Rehabilitation eine positive Tendenz ohne statistische Signifikanz, nach 6 und 12 Monaten findet sich für beide Gruppen eine eher verschlechterte Einschätzung der Lebensqualität.

\section{Diskussion}

Trainingsprogramme für die Rehabilitation von COPD-Patienten sind inzwischen in die Empfehlungen der europäischen und amerikanischen Atemwegsgesellschaften aufgenommen [12,13]. Die Empfehlungen der deutschen Atemwegsliga zum Training bei obstruktiven Atemwegskrankheiten sind kürzlich erst neu erschienen [14]. Die Trainingsmodalitäten sind noch nicht standardisiert, jedoch scheinen sich sportmedizinisch orientierte Konzepte durchzusetzen, wobei es Hinweise dafür gibt, dass insbesondere im ambulanten Bereich Patienten auch von Trainingsintensitäten unterhalb der anaeroben Schwelle profitieren $[15,16,17]$. Für Trainingsprogramme oberhalb der anaeroben Schwelle sind die sportmedizinisch erwarteten Trainingsergebnisse gesichert [5], und eine neuere Arbeit [18] belegt auch für 
Tab. 7 MOS SF-36 6 Monate/12 Monate nach stationärer Rehabilitation. Die Signifikanzen wurden berechnet zum Basiswert vor Rehabilitation

\begin{tabular}{|c|c|c|c|c|}
\hline & \multicolumn{2}{|c|}{ Kontrollgruppe } & \multicolumn{2}{|c|}{ Interventionsgruppe } \\
\hline & $\begin{array}{l}n=36 \\
6 \text { Monate }\end{array}$ & $\begin{array}{l}n=34 \\
12 \text { Monate }\end{array}$ & $\begin{array}{l}n=69 \\
6 \text { Monate }\end{array}$ & $\begin{array}{l}n=60 \\
12 \text { Monate }\end{array}$ \\
\hline $\begin{array}{l}\text { körperliche Funk- } \\
\text { tionsfähigkeit }\end{array}$ & $64,7 \pm 24,6$ & $65,0 \pm 24,9$ & $71,2 \pm 22,8$ & $69,3 \pm 22,2$ \\
\hline $\begin{array}{l}\text { körperliche Rollen- } \\
\text { funktion }\end{array}$ & $47,2 \pm 47,0$ & $35,6 \pm 41,0$ & $59,6 \pm 42,4^{* *}$ & $56,7 \pm 45,1$ \\
\hline $\begin{array}{l}\text { körperliche } \\
\text { Schmerzen }\end{array}$ & $60,1 \pm 29,2$ & $56,8 \pm 25,1$ & $61,6 \pm 31,5$ & $61,5 \pm 30,3$ \\
\hline $\begin{array}{l}\text { allgemeine Gesund- } \\
\text { heitswahrnehmung }\end{array}$ & $47,5 \pm 17,3$ & $44,1 \pm 18,2^{*}$ & $46,2 \pm 20,9$ & $43,2 \pm 22,5$ \\
\hline Vitalität & $49,9 \pm 22,6$ & $47,2 \pm 17,2$ & $51,1 \pm 21,1$ & $46,9 \pm 20,3$ \\
\hline $\begin{array}{l}\text { soziale Funktions- } \\
\text { fähigkeit }\end{array}$ & $46,9 \pm 11,7$ & $47,0 \pm 12,9$ & $47,2 \pm 9,5$ & $48,2 \pm 9,4$ \\
\hline $\begin{array}{l}\text { emotionale Rollen- } \\
\text { funktion }\end{array}$ & $71,4 \pm 42,9$ & $51,5 \pm 47,9$ & $69,0 \pm 42,2$ & $64,5 \pm 44,7$ \\
\hline $\begin{array}{l}\text { psychisches Wohl- } \\
\text { befinden }\end{array}$ & $65,6 \pm 18,7$ & $65,3 \pm 16,3$ & $66,1 \pm 20,0$ & $63,5 \pm 21,6$ \\
\hline $\begin{array}{l}\text { beobachtete } \\
\text { Gesundheits- } \\
\text { veränderung }\end{array}$ & $2,9 \pm 1,2$ & $3,2 \pm 0,9$ & $2,8 \pm 1,0$ & $3,3 \pm 1,0$ \\
\hline
\end{tabular}

*signifikant bei $\mathrm{p}<0,05,{ }^{*}$ signifikant bei $\mathrm{p}<0,01$

Tab. 8 Auswertung des LAQ-Fragebogens, Skalenwert 0-100

\begin{tabular}{lll}
\hline & $\begin{array}{l}\text { Kontrollgruppe } \\
\boldsymbol{n}=39\end{array}$ & $\begin{array}{l}\text { Interventionsgruppe } \\
\boldsymbol{n}=\mathbf{8 4}\end{array}$ \\
\hline vor stationärer Rehabilitation & $61,4 \pm 20, \mathrm{n}=39$ & $57,8 \pm 20, \mathrm{n}=83$ \\
\hline nach stationärer Rehabilitation & $60,2 \pm 20, \mathrm{n}=38$ & $60,6 \pm 21, \mathrm{n}=81$ \\
\hline 6 Monate nach Rehabilitation & $57,0 \pm 21^{*}, \mathrm{n}=36$ & $58,6 \pm 23, \mathrm{n}=69$ \\
\hline 12 Monate nach Rehabilitation & $56,3 \pm 20^{*}, \mathrm{n}=34$ & $55,4 \pm 23, \mathrm{n}=60$ \\
\hline
\end{tabular}

*signifikant bei $p<0,05$

COPD-Patienten eine bioptisch gesicherte Trainingsadaptation am Skelettmuskel.

Eine Metaanalyse von insgesamt 14 Trainingsstudien [19] mit mindestens 4 Wochen Dauer hat signifikante Verbesserungen sowohl bezüglich körperlicher Leistungsfähigkeit als auch bezüglich Lebensqualität ergeben, wobei der Zuwachs an Lebensqualität deutlicher war. Mehrere Arbeiten [20] verweisen darauf, dass für die Patienten $\mathrm{FEV}_{1}$ und $\mathrm{VO}_{2 \max }$ nur schwach mit dem Empfinden von Kurzluftigkeit korrelieren.

Auch die von uns gefundene trainingsbedingte Verbesserung kardiopulmonaler Parameter entspricht im Wesentlichen der sportmedizinisch erwarteten Trainingsadaptation. An primären Effekten wird erwartet:

Kardial: Ökonomisierung der Herzarbeit mit Anstieg der Maximalleistung. Metabolisch: Steigerung der oxidativen Kapazität des Muskels. Atmung: Verminderter Atemaufwand bei gegebener Belastung [21].

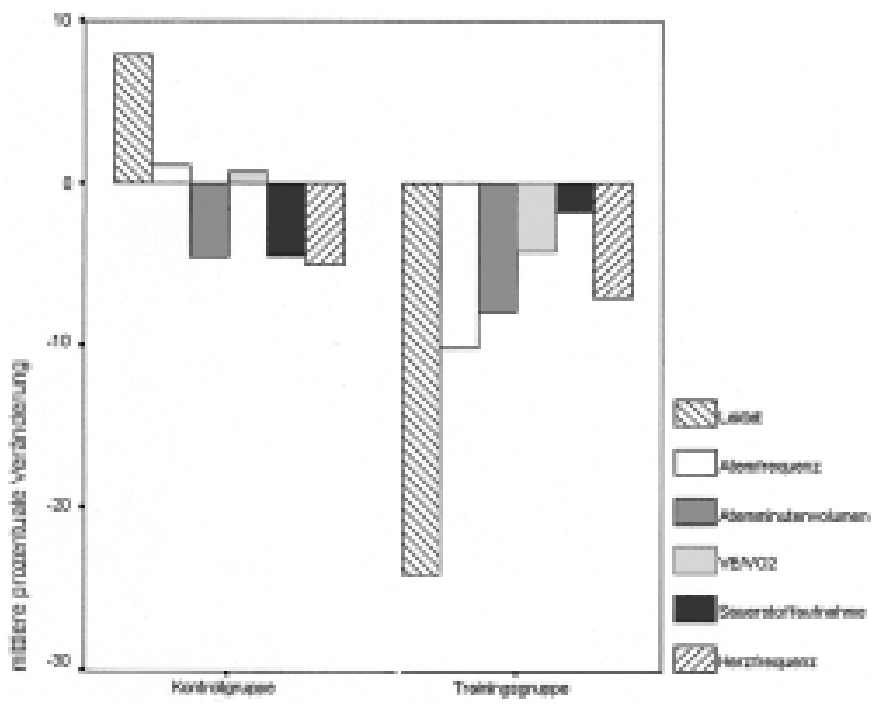

Abb. 2 Physiologische Veränderungen durch das Trainingsprogramm, prozentuale Veränderung der Werte bei identischer Belastung.

Die Ergebnisse unserer Studie sind in der folgenden Abb. 2 nochmals als prozentuale Veränderungen zusammengefasst.

Unsere Ergebnisse entsprechen prinzipiell denen der grundlegenden Arbeit von Casaburi et al. [5], dort war allerdings bei kleiner Patientenzahl ein Kontrollkollektiv nicht vorhanden, sondern es wurden zwei Interventionsgruppen mit hoher/niedriger Trainingsbelastung verglichen. In unserer Untersuchung werden durch den Vergleich mit einem nicht trainierenden Kontrollkollektiv die positiven Effekte eines Ausdauertrainings noch verdeutlicht.

Das deutsche System der stationären Rehabilitation beinhaltet neben dem Ausdauertraining auch die gesamte Bandbreite der physikalischen Therapien sowie Schulungsprogramme als Therapieprinzip. Wie Abb. 3 zeigt, haben davon und von der Optimierung der Medikation Kontroll- und Interventionsgruppe deutlich, aber ohne statistische Gruppendifferenzen profitiert. Somit

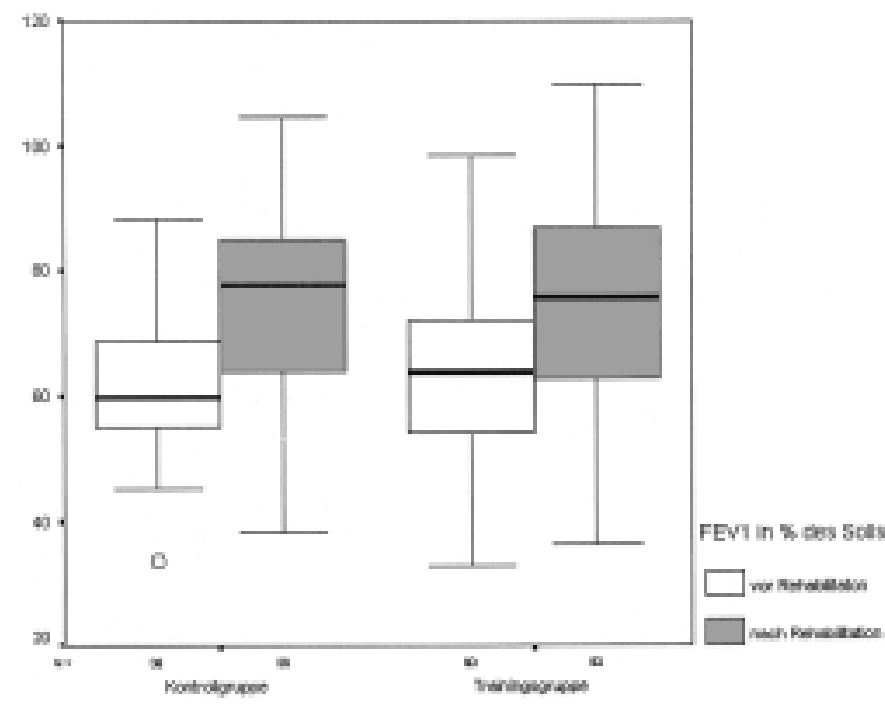

Abb.3 Veränderung der Einsekundenkapazität vor/nach stationärer Rehabilitation. 


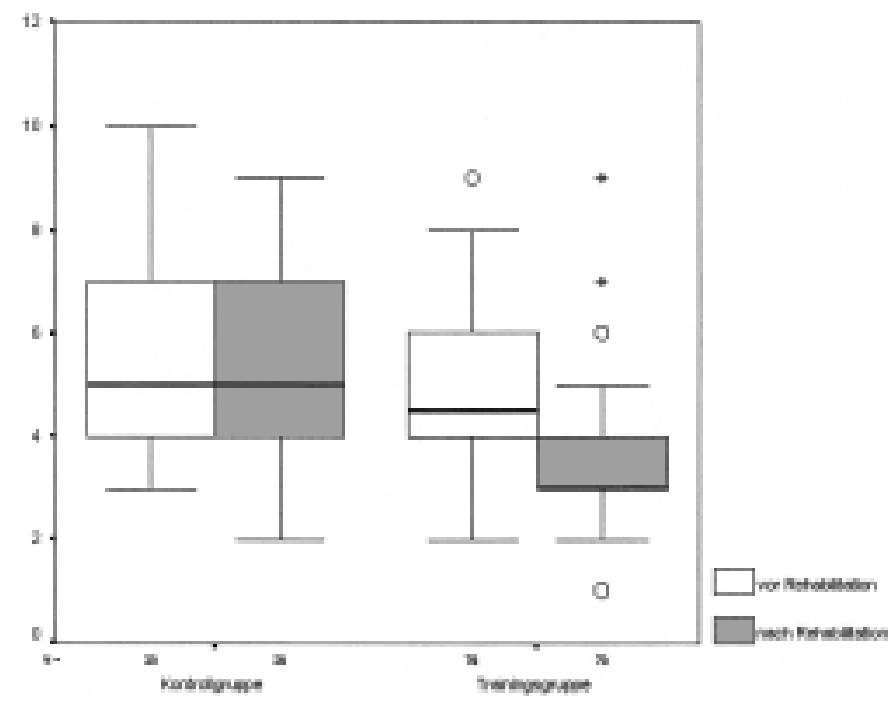

Abb. 4 Belastungsempfinden nach der modifizierten Borg-Skala. 15. Minute der Steady-State-Belastung.

sind die in der Abb. 2 dargestellten Veränderungen auch tatsächlich als Trainingseffekte zu werten.

Wie schon in mehreren Studien beschrieben, wird von COAD Patienten nach körperlichem Training eine gegebene Belastung als weniger anstrengend empfunden. Dieses wird teils auf Trainingseffekte mit Reduktion von Herzfrequenz und Atemarbeit zurückgeführt, teils wird eine Adaptation an Belastungen durch die häufigen Trainingseinheiten angenommen [19]. Entsprechend zeigt auch unsere Studie nur für die Interventionsgruppe einen deutlichen und statistisch signifikanten Abfall der BorgWerte in der Steady-State-Belastung nach Rehabilitation (Abb. 4, Tab. 5). In der Kontrollgruppe hingegen wird vor/nach Rehabilitation die subjektive Kurzluftigkeit bei gegebener Trainingsbelastung als gleich empfunden, obwohl auch hier die Einsekundenkapazität deutlich verbessert ist und die vorgegebene Belastung bei niedrigerer Herzfrequenz erbracht werden kann.

Generell wird davon ausgegangen, dass sich durch pulmonale Rehabilitation die Lebensqualität von COAD-Patienten steigern lässt [12]. Bezüglich der zu verwendenden Lebensqualitätsfragebogen herrscht kein genereller Konsens, so dass sich die Ergebnisse nur schwer vergleichen lassen. Mehrere Publikationen $[23,24,25]$ verweisen darauf, dass krankheitsspezifische Frageinstrumente wie der SGRQ oder der CRQ bezüglich der Sensitivität Vorteile bieten. Aber auch der nicht krankheitsspezifische MOS SF-36 liefert statistisch signifikante Verbesserungen in einigen Subskalen [26], wobei in unserer Studie die Interventionsgruppe deutlicher profitierte. In beiden Gruppen sind nach 6 und 12 Monaten die positiven Veränderungen wieder rückläufig. Der von uns als krankheitsspezifisches Frageinstrument gewählte LAQ-Fragebogen zeigt ausgehend von dem Basiswert vor Rehabilitation nur für die Interventionsgruppe positive Tendenzen bezüglich eines Gewinns an Lebensqualität, statistische Signifikanz wird aber nicht erreicht. In der Kontrollgruppe findet sich keine statistisch signifikante Veränderung der Befindlichkeit. 6 und 12 Monate nach der Rehabilitation kommt es in beiden Gruppen zu einer statistisch signifikanten Verschlechterung der Befindlichkeit.
Schlussfolgerungen

Es war Anliegen der Studie, das Konzept eines sportmedizinisch orientierten Trainings für COAD-Patienten an einem größeren Kollektiv während stationärer Rehabilitation zu überprüfen. Es lässt sich belegen, dass physiologische Trainingserfolge auch im Rahmen des deutschen Systems der Rehabilitation mit relativ kurzer Rehadauer zu erzielen sind. Trainingsintensitäten deutlich oberhalb der anaeroben Schwelle scheinen praktikabel und werden von den Patienten akzeptiert. Positive Erfolge der Rehabilitation schlagen sich in Teilbereichen der Fragebogen zur Lebensqualität nieder und sind 6 und 12 Monate nach Rehabilitation jeweils deutlich rückläufig. Als Fernziel ist ein System vernetzter Strukturen mit flächendeckenden ambulanten Trainingsgruppen ähnlich den Koronarsportgruppen anzustreben, wie es auch in den Empfehlungen der deutschen Atemwegsliga [14] beschrieben wird.

\section{Literatur}

${ }^{1}$ Donner CF, Muir JF. Selection criteria and programmes for pulmonary rehabilitation in COPD patients. Eur Respir J 1997; 10: 744-757

2 Patessio A, Casaburi R, Prefaut C, Folgering H, Donner C. Excercise training in chronic lung disease: exercise prescription. Eur Respir Mon 1997; 6: 129-140

${ }^{3}$ Hollmann W, Hettinger Th. Sportmedizin, Arbeits- und Trainingsgrundlagen. Schattauer, 1990: 476-483

${ }^{4}$ Gosselink R, Troosters T, Decramer M. Exercise training in COPD patients: the basic questions. Eur Respir J 1997; 10: 2884-2891

${ }^{5}$ Casaburi R, Patessio A, Ioli F, Zanaboni S, Donner CF, Wassermann K. Reductions in exercise lactic acidosis and ventilations as a result of exercise training in patients with chronic obstructive lung disease. Am Rev Resp Dis 1991; 143: 9-18

${ }^{6}$ Carter R, Coast R, Idell S. Exercise training with chronic obstructive pulmonary disease. Medicine and Science in Sports and Exercise 1992; 24: 3: $281-291$

${ }^{7}$ Behnke M, Taube C, Kirsten D, Lehnigk B, Jörres RA, Magnussen H. Long term effects of home-based exercise training in patients with severe COPD. Am J Respir Crit Care Med 1999; 159: A477

8 Baum M, Liesen H. Sport und Immunsystem. Dt Ärztebl 1998; 95 [Heft 10]: A-538-541

${ }^{9}$ Dickhut H.-H, Löllgen H. Trainingsberatung für Sporttreibende. Deutsches Ärzteblatt 1996; 93: 939-943

${ }^{10}$ Borg GAV. Psychophysical bases of perceived exertion. Med Sci Sports Exerc 1982; 14: 377-381

${ }^{11}$ Muehlig S, Petermann F. Krankheitsspezifische Erhebungsverfahren zur Lebensqualität bei Patienten mit Asthma und chronisch-obstruktiver Bronchitis. Rehabilitation 1998 Aug; 37; 3: 25-28

12 ERS Consensus Statement. Optimal assessment and management of chronic obstructive pulmonary disease. Eur Respir Rev 1996; 6: 39: $270-275$

${ }^{13}$ Pulmonary Rehabilitation. Joint ACCP/AACVPR Evidence-Bases Guidelines. Chest 1997; 112: $1363-1396$

${ }^{14}$ Empfehlungen der Deutschen Atemwegsliga zum Sport und körperlichen Training bei Patienten mit obstruktiven Atemwegserkrankungen. Pneumologie 2000; 54: $61-67$

15 Wijkstra PJ, Van der Mark TW, Kraan J, Van Altena R, Koeter GH, Postma DS. Long-term effects of home rehabilitation on physical performance in chronic obstructive pulmonary disease. Am J Respir Crit Care Med 1996; 153: 1234-1241

${ }^{16}$ Wijkstra PJ, Ten Vergert EM, Van Altena R, Otten V, Kraan J, Postma DS, Koeter GH. Long term benefits of rehabilitation at home on quality of life and exercise tolerance in patients with chronic obstructive pulmonary disease. Thorax 1995; 50: 824-828

${ }^{17}$ Clark CJ, Cochrane L, Mackay E. Low intensity peripheral muscle conditioning improves exercise tolerance and breathlessness in COPD. Eur Resp J 1996 Dec; 9705; 9; 12: 2590-2596

${ }^{18}$ Maltais F, Le Blanc P, Simard C, Jobin J, Berube C, Bruneau J, Carrier L, Belleau R. Skeletal muscle adaptation in patients with chronic ob- 
structive pulmonary disease. Am J Respir Crit Care Med 1996; 154; 2 Pt 1: $442-447$

${ }^{19}$ Lacasse Y, Wong E, Guyatt GH, King D, Cook DJ, Goldstein RS. Metaanalysis of respiratory rehabilitation in chronic obstructive pulmonary disease. Lancet 1996 Oct 26; 9702; 348; 9035: 1115-1119

${ }^{20}$ Chest Editorials. Is there really a controversy surrounding the effectiveness of respiratory rehabilitation in COPD? Chest July 1998; 114: 1

${ }^{21}$ Casaburi R. Exercise Training in Chronic Obstructive Lung Disease. In Principles and Practice of Pulmonary Rehabilitation. Saunders, 1993: 204-224

${ }^{22}$ Patessio A. Exercise training in lung disease. Eur Respir Mon 2000; 13 : 90-98

${ }^{23}$ Juniper E, Guyatt G, Ferrie P, Griffith L. Measuring Quality of Life in Asthma. Am Rev Respir Dis 1993; 147: 832 - 838

${ }^{24}$ Bergmann KCh. Asthma und Lebensqualität. Präv.-Rehab., Jahrgang 6 1994; 4: 154-158

${ }^{25}$ Donner CF, Carone M, Bertolotti G, Zotti AM. Methods of assessment of quality of life. European Respiratory Review 1997; 7: 67-80

${ }^{26}$ Boueri FMV, Bucher-Bartelson BL, Glenn KA, Make BJ. Quality of Life Measured With a Generic Instrument (Short Form-36) Improves Following Pulmonary Rehabilitation in Patients with COPD. Chest 2001; 119: 77-84

\section{Nachrichten}

Dr. med. Horst Müsken übernimmt Kuratoriumsvorsitz im Allergie-Dokumentations- und Informationszentrum (ADIZ) Bad Lippspringe

Das Allergie-Dokumentations- und Informationszentrum (ADIZ) Bad Lippspringe steht seit Beginn des Jahres 2002 unter der Leitung des Lungenfacharztes und Allergologen Dr. med. Horst Müsken. Dr. Müsken übernimmt den Vorsitz des wissenschaftlichen Kuratoriums im Informations- und Schulungszentrum von Prof. Dr. med. Karl-Christian Bergmann, der mit dem ADIZ 1991 die europaweit erste und einzige Institution dieser Art eröffnete.

In den vergangenen zehn Jahren konnte sich das ADIZ zu einer der ersten Adressen auf dem Gebiet der Allergieinformation entwickeln. Zum Aufgabengebiet gehören heute unter anderem die Enwicklung und Durchführung von Aufklärungsmaßnahmen für Betroffene auf dem Gebiet der Allergien und Atemwegserkrankungen, die Beratung von Betroffenen vor Ort und über die modernen Kommunikationsmedien und die Fort- und Weiterbildungen für Fachpersonal aus den Bereichen Medizin, Krankenpflege und Pharmazie.

Mit dem Wechsel von Dr. Müsken an die Spitze des Kuratoriums wird eine reibungslose und erfolgreiche Weiterführung der Aufklärungs- und Informationsarbeit des ADIZ gesichert, da Dr. Müsken - seit vielen Jahren Oberarzt der Bad Lippspringer Allergieund Asthmaklinik - schon seit den „Kindertagen“ des ADIZ Hand in Hand mit Prof. Bergmann die Entwicklung und Profilierung des Zentrums bis hin zur Mitarbeit im Aktionsbündnis Allergieprävention (abap) des Bundesgesundheitsministeriums begleitet. Weitere Informationen zum ADIZ und zum Programm 2002 erhalten Sie im ADIZ, Burgstr. 12, 33175 Bad Lippspringe, Tel. 05252/954502 oder www.adiz.de. 